\title{
THE STABILITY OF WEIGHTED LEBESGUE SPACES
}

\author{
BY \\ R. E. EDWARDS
}

0 . Introduction and summary. The original and principal problem studied in this paper is that of finding conditions to be satisfied by a positive "weight function" $w$ on a locally compact group in order that the set of functions $f$ such that $f^{p} w$ is integrable (relative to left Haar measure) shall be stable under left (or right) translations. Related to this is the question which asks when the set of $f$ such that $f w$ is integrable shall form an algebra under convolution.

A good deal of the preliminary matter related to the first problem can and will be formulated in a more general setting. Accordingly throughout this paper, and subject to specialization adopted in various sections, $X$ denotes a locally compact space and $\mu \neq 0$ a fixed positive Radon measure on $X . S$ is a set of homeomorphisms $s: x \rightarrow s \cdot x$ of $X$ onto itself. For our purposes there is no generality lost by assuming that $S$ is a semigroup under composition and that it contains the identity map $1: X \rightarrow X$. We assume that $\mu$ is relatively invariant under $S$, i.e. that there exists a real-valued function $\Delta$ on $S$ such that

$$
\int(f \circ s) d \mu=\Delta(s) \cdot \int f d \mu
$$

for $s \in S$ and $f \in \mathscr{K}(X)$ (the set of continuous functions on $X$ having compact supports), or, equivalently, that

$$
\mu\left(s^{-1} E\right)=\Delta(s) \cdot \mu(E)
$$

for $s \in S$ and each $\mu$-integrable set $E \subset X\left({ }^{1}\right)$. The "modular function" $\Delta$ is necessarily a strictly positive character of $S$ such that $\Delta(1)=1$.

In addition to this we fix upon a positive, locally $\mu$-integrable "weight function" $w$ which, without loss of generality, we assume to be everywhere finite. For each index $p, 1 \leqq p<+\infty$, let $\mathscr{L}^{p}(w)=\mathfrak{L}^{p}(w ; \mu)$ (resp. $\overline{\mathscr{L}}^{p}(w)$ $=\overline{\mathfrak{L}}^{p}(w ; \mu)$ ) denote the set of (finite) real-valued $\mu$-measurable functions $f$ on $X$ such that $|f|^{p_{w}}$ is $\mu$-integrable (resp. essentially $\mu$-integrable). Except in special cases (e.g. if the measure space $(X, \mu)$ is $\sigma$-finite, or if $X$ is discrete), $\mathfrak{L}^{p}(w)$ and $\overline{\mathscr{L}}^{p}(w)$ are distinct and there is a bifurcation in the original problem. However, stability of $\mathfrak{L}^{p}(w)$ is usually implied by that of $\overline{\mathfrak{L}}^{p}(w)$, and we

Received by the editors October 10, 1958.

(1) It is in fact easily shown that from (0.1) follows $\mu^{*}(f \circ s)=\Delta(s) \mu^{*}(f)$ for any function $f \geqq 0$ (finite or not), and therefore also $\bar{\mu}^{*}(f)=\Delta(s) \bar{\mu}^{*}(f)$ for any $f \geqq 0$ (finite or not). 
shall concentrate on the latter; and in any case the two problems are equivalent whenever $w>0$ a.e. $(\mu)$, which is the case most interesting in practice $\left({ }^{2}\right)$.

Temporarily disregarding the case in which $w$ admits a non-negligible set of zeros, the conditions for stability prove to be (roughly speaking) that both $w$ and $w^{-1}$ have a moderate rate of growth governed largely by that typical of the positive subcharacters of $S$.

It will be seen at the outset of $\S 1$ that the case $p=1$ governs all the rest and we subsequently take this single value of $p\left({ }^{3}\right)$.

In the absence of any statement to the contrary, the appropriate terminology and results regarding integration are taken from $[\mathrm{B}]$ and $\left[\mathrm{B}^{\prime}\right]$. In particular, if $f$ is an essentially $\mu$-integrable function, we write $\int f d \mu$ for the essential integral $\int^{-*} f d \mu\left[\mathrm{B}^{\prime}\right.$, p. 14]. The abbreviations "a.e." and "l.a.e." are used for "almost everywhere" and "locally almost everywhere" respectively. Although we shall sometimes be using several measures at once, the above phrases and others like them, if left unqualified as regards the measure in question, are to be taken to refer to $\mu$. For subsets of $X$ the term "bounded" means "relatively compact."

It rarely happens that any one of the propositions to follow requires all the assumptions made. However, for the sake of brevity, we leave aside any discussion of a minimal set of hypotheses.

1. The main theorem. We begin by recording two simple results, neither of which requires formal proof.

1.1. If $1 \leqq p<+\infty, \overline{\mathscr{L}}^{p}(w)$ (resp. $\mathfrak{L}^{p}(w)$ ) is stable under $S$ if and only if $\overline{\mathfrak{L}}^{1}(w)$ (resp. $\mathfrak{L}^{1}(w)$ ) is stable under $S$.

Accordingly we assume henceforth that $p=1$.

1.2. If $w=0$ l.a.e., then both $\overline{\mathcal{L}}^{1}(w)$ and $\mathfrak{L}^{1}(w)$ are stable under $S$.

We now work towards Proposition 1.5 below. For this it is necessary to introduce the unique decomposition

$$
\mu=\mu_{1}+\mu_{2}
$$

with $\mu_{1}$ diffuse and $\mu_{2}$ atomic [B', p. 61, Définitions 5, 6]; $\mu_{1}$ and $\mu_{2}$ are necessarily estranged so that $\left[\mathrm{B}^{\prime}\right.$, p. 53] there exist disjoint subsets $M_{1}, M_{2}$ of $X$ such that $M_{i}^{\prime}$ is locally $\mu_{i}$-negligible $(i=1,2)$. We are here writing $M^{\prime}$ for the complement, relative to $X$, of $M$. The explicit formula for the atomic part of $\mu$ is

$$
\mu_{2}=\sum_{x \in X} \mu(\{x\}) \cdot \epsilon_{x},
$$

where $\boldsymbol{\epsilon}_{x}$ is the Dirac measure at $x$; the series is unconditionally convergent in the sense of the vague topology on the set of measures. Equation (1.2) shows that, if $\mu$ is relatively invariant, the same is true of $\mu_{2}$ and hence of $\mu_{1}$ too. So in this case $s^{-1} M_{i}^{\prime}$ is locally $\mu_{i}$-negligible for each $s \in S$ and $i=1,2$.

(2) See 1.7 and 1.8 .

(3) See 1.1. 
1.3. Proposition. $\overline{\mathfrak{L}}^{1}(w ; \mu)$ is stable under $S$ if and only if the same is true of $\overline{\mathfrak{L}}^{1}\left(w ; \mu_{i}\right)(i=1,2)$.

Proof. The relation

$$
\overline{\mathfrak{L}}^{1}(w ; \mu)=\overline{\mathfrak{L}}^{1}\left(w ; \mu_{1}\right) \cap \overline{\mathfrak{L}}^{1}\left(w ; \mu_{2}\right)
$$

shows at once that the stability of both $\overline{\mathscr{L}}^{1}\left(w ; \mu_{i}\right)$ entails that of $\overline{\mathscr{L}}^{1}(w ; \mu)$. Conversely, suppose that $\overline{\mathscr{L}}^{1}(w ; \mu)$ is stable. If $f \in \overline{\mathscr{L}}^{1}\left(w ; \mu_{1}\right)$, define $f_{1}$ to be equal to $f$ on $M_{1}$ and to be 0 elsewhere. Since $M_{1}^{\prime} \supset M_{2}$ and $M_{i}^{\prime}$ is locally negligible for $\mu_{i}$, it follows that $f_{1}$ belongs to $\overline{\mathscr{L}}^{1}\left(w ; \mu_{i}\right)$ for $i=1,2$. Thus $f_{1} \in \overline{\mathcal{L}}^{1}(w ; \mu)$. By hypothesis, therefore, $f_{1} \circ s \in \overline{\mathcal{L}}^{1}(w ; \mu) C \overline{\mathscr{L}}^{1}\left(w ; \mu_{1}\right)$ for each $s \in S$. But, since $s^{-1} M_{1}^{\prime}$ is locally negligible for $\mu_{1}, f \circ s=f_{1} \circ s$ l.a.e. $\left(\mu_{1}\right)$; so $f \circ s \in \overline{\mathscr{L}}^{1}\left(w ; \mu_{1}\right)$. This shows that $\overline{\mathscr{L}}^{1}\left(w ; \mu_{1}\right)$ is stable. Similarly for $\overline{\mathscr{L}}^{1}\left(w ; \mu_{2}\right)$. This completes the proof.

We shall say that a positive measure $\nu$ has the property $\left(^{*}\right)$ relative to $S$ if, whenever $A$ and $B$ are bounded $\nu$-integrable sets such that $\nu(A)>0$ and $\nu(B)>0$, there exists $s \in S$ such that $\nu\left(A \cap s^{-1} B\right)>0$.

\subsection{Lemma. If $\mu$ has property $\left({ }^{*}\right)$, so have both $\mu_{1}$ and $\mu_{2}$.}

Proof. It suffices to show that $\mu_{1}$ has property $(*)$, the proof for $\mu_{2}$ being entirely similar. Let $A$ and $B$ be bounded $\mu_{1}$-integrable sets such that $\mu_{1}(A)>0$ and $\mu_{1}(B)>0$. Since $M_{1}^{\prime}$ locally negligible for $\mu_{1}$, we may assume that $A \subset M_{1}$ and $B C M_{1}$-if not, replace them by their intersections with $M_{1}$. Since $M_{1} \subset M_{2}^{\prime}$ is locally $\mu_{2}$-negligible, the same is true of $A$ and $B$. Hence $A$ and $B$ are $\mu$-integrable. Further, since

$$
\mu(A)=\mu_{1}(A)>0
$$

and likewise $\mu(B)>0$, it follows that there exists $s \in S$ such that $\mu\left(A \cap s^{-1} B\right)$ $>0$. However,

$$
\mu_{1}\left(A \cap s^{-1} B\right)=\mu\left(A \cap s^{-1} B\right)
$$

since $A$ is $\mu_{2}$-negligible. Thus $\mu_{1}$ has property $\left({ }^{*}\right)$.

1.5. Proposition. Suppose that $\mu$ has property $\left(^{*}\right)$ and that $f \circ s \in \overline{\mathfrak{L}}^{1}(w ; \mu)$ whenever $f \in \mathcal{L}^{1}(w ; \mu)$ has a bounded support. Then either (i) $w=0$ l.a.e. $\left(\mu_{1}\right)$ or (ii) $w>0$ l.a.e. $\left(\mu_{1}\right)$.

Proof. The proof of Proposition 1.3 shows that the stability hypothesis remains fulfilled if therein we replace $\mu$ by $\mu_{1}$. This, together with Lemma 1.4, shows that we may assume throughout that $\mu$ is diffuse.

In this case, and assuming (ii) to be false, there is a compact set $A$ such that $\mu(A)>0$ and $w=0$ on $A$. Then any $\mu$-measurable function $f$ which vanishes on $A^{\prime}$ will belong to $\mathscr{L}^{1}(w ; \mu)$. This, together with the main hypothesis, shows that 


$$
\int_{s^{-1} A} g w d \mu<+\infty
$$

for every $\mu$-measurable $g \geqq 0$. From this we may conclude that $w=0$ a.e. on $s^{-1} A$ : indeed, in the contrary case, there would exist a compact $B \subset s^{-1} A$ with $\mu(B)>0$ and $w \geqq c>0$ on $B$ ( $c$ a number). Since $\mu$ is diffuse we can [B', p. 63, Exercise 9, b] find a sequence $\left(B_{n}\right)$ of disjoint integrable subsets of $B$ such that $\mu\left(B_{n}\right)>0$ for each $n$. If we define $g=\mu\left(B_{n}\right)^{-1}$ on $B_{n}(n=1,2, \cdots)$ and $g=0$ elsewhere, we get

$$
\int_{s^{-1} A} g w d \mu=\sum_{n=1}^{\infty} \int_{B_{n}} g w d \mu \geqq \sum_{n=1}^{\infty} c \cdot \mu\left(B_{n}\right)^{-1} \cdot \mu\left(B_{n}\right)=+\infty,
$$

a contradiction. Thus $w=0$ a.e. on $s^{-1} A$, and this for each $s \in S$. If therefore $C$ is any $\mu$-integrable set with $\mu(C)>0$, and if we choose $s$ so that $\mu\left(C \cap s^{-1} A\right)$ $>0$ (as we may by $\left({ }^{*}\right)$ ), we see that $C$ contains a subset $C^{\prime}=C \cap s^{-1} A$ such that $\mu\left(C^{\prime}\right)>0$ and $w=0$ a.e. on $C^{\prime}$. In other words, every compact $C$ with $\mu(C)>0$ contains a $C^{\prime \prime}$ with $\mu\left(C^{\prime \prime}\right)>0$ such that $w=0$ on $C^{\prime \prime}$. Thanks to the arbitrary nature of $C$, this shows that $w=0$ l.a.e. This completes the proof.

1.6. Corollary. If $\mu$ is diffuse and satisfies $\left({ }^{*}\right)$, and if either of $\overline{\mathfrak{L}}^{1}(w ; \mu)$ or $\mathfrak{L}^{1}(w ; \mu)$ is stable under $S$, then either $w=0$ l.a.e. or $w>0$ l.a.e.

The next proposition deals with the relationship between the stability of the two spaces in question.

1.7. Proposition. Let $P=\{x \in X: w(x)>0\}$.

(a) If $P \cap s^{-1} P^{\prime}$ is locally negligible for each $s \in S$, stability of $\mathscr{L}^{1}(w ; \mu)$ implies that of $\overline{\mathfrak{L}}^{1}(w ; \mu)$.

(b) If $P \cap s^{-1} P^{\prime}$ is $\sigma$-finite for each $s \in S$, stability of $\overline{\mathcal{L}}^{1}(w ; \mu)$ implies that of $\mathcal{L}^{1}(w ; \mu)$.

Proof. (a) Let $f \in \overline{\mathscr{L}}^{1}(w ; \mu)$. Then there exists $h \in \mathcal{L}^{1}(\mu)$ such that $f w=h$ 1.a.e. Define $g$ to be equal to $h w^{-1}$ on $P$ and to 0 on $P^{\prime}$. Then $g$ is measurable and $|g| w \leqq|h|$ and so $g \in \mathscr{L}^{1}(w ; \mu)$. Also $g=f$ l.a.e. on $P$. Since $\mathscr{L}^{1}(w ; \mu)$ is stable (by hypothesis), $g \circ s \in \mathscr{L}^{1}(w ; \mu)$. However, by relative invariance, the inverse image by $s$ of any locally negligible set is again locally negligible. So $f \circ s=g \circ s$ 1.a.e. on $s^{-1} P$ and $(f \circ s) w=(g \circ s) w$ l.a.e. on $P^{\prime} \cup s^{-1} P$. Since $\left(P^{\prime} \cup s^{-1} P\right)^{\prime}=P \cap s^{-1} P^{\prime}$ is locally negligible, it follows that $f \circ s \in \overline{\mathcal{L}}^{1}(w ; \mu)$. Thus $\overline{\mathscr{L}}^{1}(w ; \mu)$ is stable.

(b) It is clear that $\mathscr{L}^{1}(w ; \mu)=\overline{\mathscr{L}}^{1}(w ; \mu) \cap \mathcal{F}$, where $\mathcal{F}$ is the set of functions $f$ such that $f w$ vanishes outside a $\sigma$-finite set. Hence, given the stability of $\overline{\mathcal{L}}^{1}(w ; \mu)$, that of $\mathcal{L}^{1}(w ; \mu)$ follows from that of $\mathcal{F}$. To investigate the stability of $\mathcal{F}$, take any $f \in \mathcal{F}$. Then $f w=0$ on $Q^{\prime}$, where $Q$ is $\sigma$-finite. Hence $f=0$ on $P \cap Q^{\prime}, f \circ s=0$ on $s^{-1}\left(P \cap Q^{\prime}\right)=\left(s^{-1} P\right) \cap\left(s^{-1} Q\right)^{\prime}$ and so $(f \circ s) w=0$ on $P^{\prime} \cup\left[\left(s^{-1} P\right) \cap\left(s^{-1} Q\right)^{\prime}\right]=R^{\prime}$, where 


$$
R=\left(P \cap s^{-1} P^{\prime}\right) \cup\left(P \cap s^{-1} Q\right) .
$$

Now $R$ is $\sigma$-finite together with $P \cap s^{-1} P^{\prime}$. Whence the assertion (b).

1.8. Corollary. (a) If either $P$ or $P^{\prime}$ is locally negligible, stability of $\mathfrak{L}^{1}(w ; \mu)$ implies that of $\overline{\mathfrak{L}}^{1}(w ; \mu)$.

(b) If either $P$ or $P^{\prime}$ is $\sigma$-finite, stability of $\overline{\mathfrak{L}}^{1}(w ; \mu)$ implies that of $\mathfrak{L}^{1}(w ; \mu)$.

(c) If $\mu$ is diff use and satisfies $\left({ }^{*}\right)$, stability of $\mathscr{L}^{1}(w ; \mu)$ implies that of $\overline{\mathfrak{L}}^{1}(w ; \mu)$.

(d) Stability of $\bar{L}^{1}(w ; \mu)$ is equivalent to that of $\mathscr{L}^{1}(w ; \mu)$ in any one of the following cases:

(i) either $w=0$ a.e. or $w>0$ a.e.;

(ii) $X$ is $\sigma$-finite;

(iii) $X$ is discrete;

(iv) $\mu$ is diffuse and satisfies $\left({ }^{*}\right)$, and either $P$ or $P^{\prime}$ is $\sigma$-finite.

1.10. Remark. One is not at liberty to assume that $S$ is a full group: stability under $s$ does not imply stability under $s^{-1}$. Example: Take $X$ to be the real axis, $\mu$ to be the Lebesgue measure, and define $s \cdot x=s x$ (usual product). We suppose $s>0$. Relative invariance is clear, with $\Delta(s)=s^{-1}$. For $w(x)$ take $\exp (|x|)$. Since

$$
\int_{-\infty}^{\infty}|f(s x)| \exp (|x|) d x=s^{-1} \int_{-\infty}^{\infty}|f(x)| \exp \left(s^{-1}|x|\right) d x
$$

we see that $\mathcal{L}^{1}(w ; \mu)$ (here identical with $\overline{\mathfrak{L}}^{1}(w ; \mu)$ ) is stable under $x \rightarrow s x$ if and only if $s \geqq 1$. Thus if we suppose $s>1$, stability obtains for $x \rightarrow s x$ but not for the inverse map $x \rightarrow s^{-1} x$.

We pass now to a proposition which contains the kernel of the main theorem.

1.11. Proposition. Suppose that for a certain $s \in S$ the set $P \cap s^{-1} P^{\prime}$ is locally negligible, and that the mapping $f \rightarrow f \circ s$ leaves $\overline{\mathscr{L}}^{1}(w ; \mu)$ stable. Then there exists a number $M \geqq 0$ such that

$$
\int|f \circ s| w d \mu \leqq M \cdot \int|f| w d \mu
$$

for $f \in \overline{\mathcal{L}}^{1}(w ; \mu)\left({ }^{4}\right)$.

Proof. Introduce the seminorm on $\overline{\mathscr{L}}^{-1}(w ; \mu)$ defined by

$$
N(f)=\int|f| w d \mu
$$

( $\left.{ }^{4}\right)$ There is an analogous result for $\mathcal{L}^{1}(w ; \mu)$. 
which defines a (generally nonseparated) uniform structure and associated topology. By hypothesis,

$$
N^{\prime}(f)=\int|f \circ s| w d \mu
$$

is a second seminorm on $\overline{\mathscr{L}}^{1}(w ; \mu)$. Our task is prove that $N^{\prime}$ is continuous relative to $N$. We shall show first that $\overline{\mathcal{L}}^{1}(w ; \mu)$ is complete, hence a Baire space. When this is done, Baire's theorem( $\left.{ }^{5}\right)$ shows that continuity of $N^{\prime}$ follows from lower semicontinuity. Accordingly the proof falls into two parts.

(1) Completeness of $\overline{\mathscr{L}}^{1}(w ; \mu)$. This does not depend on any hypothesis concerning $P$. Let $\left(f_{n}\right)$ be a Cauchy sequence in $\overline{\mathscr{L}}^{1}(w ; \mu)$. There is then a $\sigma$-finite set $T \subset X$ and a locally negligible set $T^{*} \subset X$ such that all the $f_{n}$ vanish outside $T \cup T^{*}$. Then, if $P$ is the set $\{x \in X: w(x)>0\}$, we have

$$
\int\left|f_{i}-f_{j}\right| w d \mu=\int_{P \cap T}\left|f_{i}-f_{j}\right| w d \mu .
$$

A familiar argument now shows that the Cauchy character of $\left(f_{n}\right)$ entails the existence of a subsequence $\left(f_{n_{k}}\right)$ which converges a.e. on $P \cap T$ to a measurable function $f$. Extend $f$ by setting it equal to zero outside $P \cap T$. Then $f_{n_{k}} w \rightarrow f w$ holds 1.a.e. A modified form of Fatou's lemma [ $\mathrm{B}^{\prime}$, p. 16, Exercise 1] shows that therefore

$$
\int|f| w d \mu \leqq \liminf _{k \rightarrow \infty} \int\left|f_{n_{k}}\right| w d \mu<+\infty,
$$

so that $f \in \overline{\mathscr{L}}^{1}(w ; \mu)$. Likewise

$$
\int\left|f-f_{n}\right| w d \mu \leqq \liminf _{k \rightarrow \infty} \int\left|f_{n_{k}}-f_{n}\right| w d \mu,
$$

which tends to zero as $n \rightarrow \infty$. This $f$ is thus a limit in $\overline{\mathcal{L}}^{1}(w ; \mu)$ of the sequence $\left(f_{n}\right) \cdot \overline{\mathfrak{L}}^{1}(w ; \mu)$ is thereby shown to be complete.

(2) Lower semicontinuity of $N^{\prime}$. It suffices to show that the set $\mathfrak{T} \subset \overline{\mathscr{L}}^{1}(w ; \mu)$, comprising those $f \in \overline{\mathscr{L}}^{1}(w ; \mu)$ satisfying $N^{\prime}(f) \leqq 1$, is closed in $\overline{\mathscr{L}}^{1}(w ; \mu)$. However, if $\left(f_{n}\right)$ is a sequence extracted from $\mathscr{T}$ which converges in $\overline{\mathscr{L}}^{1}(w ; \mu)$ to $f$, we may (as above) extract a subsequence $\left(f_{n_{k}}\right)$ such that $f_{n_{k}} w \rightarrow f w$ on $E^{\prime}$, where $E$ is locally negligible. This shows that $f_{n_{k}} \rightarrow f$ on $P \cap E^{\prime}$, hence $f_{n_{k}} \circ \mathrm{s}$ $\rightarrow f \circ s$ on $s^{-1}\left(P \cap E^{\prime}\right)$, and so $\left(f_{n_{k}} \circ s\right) w \rightarrow(f \circ s) w$ on $P^{\prime} \cup s^{-1}\left(P \cap E^{\prime}\right)$, i.e. on $R^{\prime}$ where $R=\left(P \cap s^{-1} P^{\prime}\right) \cup\left(P \cap s^{-1} E\right) . R$ is locally negligible or not together with $P \cap s^{-1} P^{\prime}$. In the present instance, therefore, $\left(f_{n_{k}} \circ s\right) w \rightarrow(f \circ s) w$ l.a.e.

(5) By this we mean the conjunction of the two propositions: (a) Every complete semimetrisable space is a Baire space; (b) Every finite lower semicontinuous function on a Baire space is bounded above on some nonempty open set. See Bourbaki, Topologie générale, Chapter IX, p. 76, Théorème 1; p. 77, ThEorème 2. 
and Fatou's lemma shows that

$$
\begin{aligned}
N^{\prime}(f) & =\int|f \circ s| w d \mu \leqq \liminf _{\boldsymbol{k} \rightarrow \infty} \int\left|f_{n_{k}} \circ s\right| w d \mu \\
& =\liminf _{\boldsymbol{k} \rightarrow \infty} N^{\prime}\left(f_{n_{k}}\right) \leqq \underset{\boldsymbol{k}}{\liminf } 1=1 .
\end{aligned}
$$

Thus $f \in \mathscr{M}$ and $\mathscr{M}$ is closed. This completes the proof of Proposition 1.11.

The road to the principal theorem is now open. It will be convenient to denote by $\mathcal{E}$ the set of all bounded, integrable subsets of $X$, and then to frame a definition.

1.12. Definition. A positive locally integrable function $w$ on $X$ is said to be moderate (with respect to $S$ and $\mu$ ) if to each $s \in S$ corresponds a number $M(s) \geqq 0$ such that

$$
\int_{8^{-1} E} w d \mu \leqq M(s) \int_{E} w d \mu \quad(E \in \mathcal{E})
$$

or, what is equivalent,

$$
w\left(s^{-1} \cdot x\right) \leqq M(s) \Delta(s)^{-1} w(x) \quad \text { l.a.e., }
$$

or again

$$
\int|f \circ s| w d \mu \leqq M(s) \int|f| w d \mu \quad\left(f \in \overline{\mathscr{L}}^{1}(w ; \mu)\right) .
$$

Regarding the equivalence of these conditions, note that since

$$
\int|f \circ s| w d \mu=\Delta(s) \int|f(x)| w\left(s^{-1} \cdot x\right) d \mu(x),
$$

it is clear that $\left(1.4^{\prime}\right)$ implies $\left(1.4^{\prime \prime}\right)$. Also, $\left(1.4^{\prime \prime}\right)$ implies (1.4) on taking $f=\phi_{E}$, the characteristic function of $E$. Again,

$$
\begin{aligned}
\int_{s^{-1} E} w d \mu & =\int w(x) \phi_{E}(s \cdot x) d \mu(x) \\
& =\Delta(s) \int w\left(s^{-1} \cdot x\right) \phi_{E}(x) d \mu(x),
\end{aligned}
$$

and so (1.4) implies that

$$
\int_{E} w\left(s^{-1} \cdot x\right) d \mu(x) \leqq M(s) \Delta(s)^{-1} \int_{E} w d \mu,
$$

whence follows $\left(1.4^{\prime}\right)$. Thus

$$
\left(1.4^{\prime}\right) \Rightarrow\left(1.4^{\prime \prime}\right) \Rightarrow(1.4) \Rightarrow\left(1.4^{\prime}\right)
$$


and the conditions are indeed equivalent.

1.13. Theorem. (i) If $w$ is moderate, then $\overline{\mathfrak{L}}^{1}(w ; \mu)$ is stable.

(ii) If $w>0$ l.a.e., and if $\overline{\mathscr{L}}^{1}(w ; \mu)$ is stable, then $w$ is moderate.

(iii) If $\mu$ is diffuse and satisfies $\left(^{*}\right), \overline{\mathscr{L}}^{1}(w ; \mu)$ is stable if and only if $w$ is moderate.

Proof. (i) is trivial in view of $\left(1.4^{\prime \prime}\right)$. (ii) follows from Proposition 1.11. (iii) follows from (i) and (ii) combined with Corollary 1.6.

1.14. Remark. We have yet to supplement Theorem 1.13 by dealing with the case in which $\mu$ is atomic and $w$ admits a set of zeros which is not locally negligible: the most important case here is that of a discrete group. This question is taken up in $\$ 2$ below. Meanwhile we proceed to list some properties of the number $M(s)$ associated with a moderate weight function $w$. Henceforth we shall agree that, whenever $w$ is moderate, $M(s)$ shall denote the smallest positive number for which the conditions (1.4)- $\left(1.4^{\prime \prime}\right)$ hold.

1.15. Proposition. Suppose $w$ is moderate. Then $M(s) \equiv 0(s \in S)$ if and only if $w$ is locally negligible. If $w$ is not locally negligible, then

$$
\begin{aligned}
& M(1)=1, \quad M(s)>0 \\
& M(s t) \leqq M(s) M(t)
\end{aligned}
$$$$
(s \in S) \text {, }
$$$$
(s, t \in S) \text {. }
$$

Proof. Obvious. Note that if $w$ is moderate, and if $\mu$ satisfies (*), the proof of Proposition 1.5 may be repeated to show that $w$, if not locally negligible, is strictly positive l.a.e.

1.16. Proposition. Suppose that $w$ is moderate and not locally negligible. If $S$ be equipped with a topology such that the mapping $(s, x) \rightarrow s^{-1} \cdot x$ is continuous, then $M(s)$ is lower semicontinuous on compact subsets of $S$, and $M(s)^{-1}$ is bounded on compact subsets. If in addition $S$ is a locally compact group, then $M(s)$ is locally bounded.

Proof. Since $w$ is not locally negligible,

$$
M(s)=\operatorname{Sup}\left\{\int_{s^{-1} E} w d \mu / \int_{E} w d \mu\right\},
$$

the supremum being taken relative to $E$ varying over the set $\mathcal{E}_{0}$ of $E \in \mathcal{E}$ such that $\int_{E} w d \mu>0$. To show that $M(s)$ is lower semicontinuous it therefore suffices to show that for a fixed $E$

$$
\int_{s^{-1} E} w d \mu=\Delta(s) \int_{E} w\left(s^{-1} \cdot x\right) d \mu(x)
$$

is continuous. Thanks to the assumptions made about continuity of $s^{-1} \cdot x$, continuity of $\Delta(s)$ is clear. So we have only to consider $\int_{E} w\left(s^{-1} \cdot x\right) d \mu(x)$. Let $H \subset S$ be compact. Since $E$ is bounded, the set 


$$
\left\{s^{-1} \cdot x: s \in H, x \in E\right\}
$$

is bounded. Hence there exists an integrable $w^{\prime}$ such that

$$
\int_{E} w\left(s^{-1} \cdot x\right) d \mu(x)=\int_{E} w^{\prime}\left(s^{-1} \cdot x\right) d \mu(x)
$$

for all $s \in H$. $w^{\prime}$ being integrable, we may for any given $\epsilon>0$ choose $f \in \mathcal{K}(X)$ such that $\int\left|w^{\prime}-f\right| d \mu \leqq \epsilon$, and then

$$
\begin{aligned}
\int_{E}\left|w^{\prime}\left(s^{-1} \cdot x\right)-f\left(s^{-1} \cdot x\right)\right| d \mu(x) & =\Delta(s)^{-1} \int_{s^{-1} E}\left|w^{\prime}-f\right| d \mu \\
& \leqq \Delta(s)^{-1} \epsilon .
\end{aligned}
$$

Now $\Delta(s)^{-1}$ is bounded for $s \in H$, and $\int_{E} f\left(s^{-1} \cdot x\right) d \mu(x)$ is clearly continuous in $s$. Hence $\int_{E} w\left(s^{-1} x\right) d \mu(x)$ is the limit, uniformly on $H$, of continuous functions, hence is itself continuous.

Being everywhere strictly positive, it now follows that $M(s)$ has on $H$ a strictly positive infimum.

Finally, if $S$ is a locally compact group, Baire's Theorem shows that $M(s)$ is bounded above on some nonempty relatively open subset of $H$, and this for any compact $H$. It follows from this (by taking $H$ to be the closure of some bounded open set) that $M(s)$ is bounded above on some nonempty open set. Then (1.6) shows that $M(s)$ is bounded above on each compact set. This completes the proof.

2. The case in which $\mu$ is atomic. If $\mu$ is atomic, $\bar{L}^{1}(w ; \mu)$ may well be stable even if $w$ is not locally negligible and yet admits a set of zeros which is also not locally negligible. In this case, the analogue of Theorem 1.13 (iii) is false, though of course parts (i) and (ii) of that theorem remain true. This section deals with the case still outstanding. Most of the arguments are similar to those used above and will be presented rapidly. We begin with some remarks about atomic measures in general; all of them are elementary but the author knows of no convenient reference.

2.1. Atomic measures. A measure $\mu$ is atomic if it is of the form

$$
\mu=\sum_{x \in X} a_{x} \epsilon_{x}
$$

the series being unconditionally convergent (i.e. convergent following the increasing directed set of finite subsets of $X$ ) in the sense of the vague topology; $\epsilon_{x}$ is the Dirac measure at $x \in X$, and $a_{x}$ is a finite numerically-valued function on $X$. The convergence just described signifies that the numerical series $\sum_{x \in X} a_{x} f(x)$ is unconditionally (i.e. absolutely) convergent for each $f \in \mathfrak{K}(X)$. This is easily seen to be equivalent to the assertion that

$$
\sum_{x \in K}\left|a_{x}\right|<+\infty
$$


for each compact $K \subset X$. It follows easily that if $\mu$ is positive, as we shall assume henceforth, then $a_{x} \geqq 0$. In fact, given $x_{0}$, choose a compact neighbourhood $K$ of $x_{0}$. The set of $x \in K$ satisfying $x \neq x_{0}$ and $a_{x} \neq 0$ is then countable, thanks to (2.2). Enumerate this set as a sequence $\left(x_{n}\right)_{n \geq 1}$. Given $\epsilon>0$, choose $n$ so that

$$
\sum_{m>n}\left|a_{x_{m}}\right| \leqq \epsilon .
$$

Let $f \in \Re_{+}(X)$ be such that $0 \leqq f \leqq 1, f=0$ on $K^{\prime}, f\left(x_{0}\right)=1, f\left(x_{m}\right)=0$ for $1 \leqq m \leqq n$. Then, since $\mu \geqq 0$, one will have

$$
\begin{aligned}
0 \leqq \mu(f) & =\sum a_{x} f(x)=a_{x_{0}}+\sum_{m \geq 1} a_{x_{m}} f\left(x_{m}\right) \\
& =a_{x_{0}}+\sum_{m>n} a_{x_{m}} f\left(x_{m}\right) \leqq a_{x_{0}}+\epsilon .
\end{aligned}
$$

Thus $a_{x_{0}} \geqq-\epsilon$. Letting $\epsilon \rightarrow 0$, this gives $a_{x_{0}} \geqq 0$.

We next prove some less trivial statements, labelled (a)-(e) below, concerning the upper and essential integrals defined by $\mu$. These assertions will refer to positive functions only, but we shall admit $+\infty$ as a possible value of the functions. We adopt the usual conventions regarding the manipulation of the symbol $+\infty$, e.g. $a+(+\infty)=+\infty$ if $0 \leqq a \leqq+\infty, a \cdot(+\infty)=+\infty$ if $0<a \leqq+\infty, 0 \cdot(+\infty)=0$, and both operations are assumed to be commutative.

(a) The equation

$$
\mu^{*}(f)=\sum_{x \in X} a_{x} f(x)
$$

holds for any positive lower semicontinuous function $f$ (finite or not).

Proof. By definition [B, p. 104, Définition 1]

$$
\mu^{*}(f)=\operatorname{Sup}\left\{\mu(k): k \in \mathfrak{K}_{+}(X), k \leqq f\right\},
$$

whence it is clear that in all cases

$$
\mu^{*}(f) \leqq \sum a_{x} f(x)
$$

In proving the reverse inequality, we first assume that $f$ is finite-valued. Take any number $p<\sum a_{x} f(x)$. There exists then a finite set $F \subset X$ such that already

$$
p<\sum_{x \in F} a_{x} f(x)
$$

Choose then any $k \in \mathscr{K}_{+}(X)$ such that $k \leqq f$ and

$$
k(y) \geqq f(y)-\left(\sum_{x \in F} a_{x}\right)^{-1} \cdot \epsilon
$$


for $y \in F, \epsilon>0$ being arbitrarily preassigned. Then

$$
p<\sum_{x \in F} a_{x} k(x)+\epsilon \leqq \mu(k)+\epsilon .
$$

Thus $\mu^{*}(f) \geqq \mu(k) \geqq p-\epsilon$. The arbitrary nature of $p$ and $\epsilon$ shows that $\mu^{*}(f)$ $\geqq \sum a_{x} f(x)$, and (2.3) is thereby established in this case.

If $f$ is not finite-valued, let $f_{n}=\operatorname{Inf}(f, n)$ for $n=1,2, \cdots$; the $f_{n}$ are finite lower semicontinuous functions which increase monotonely to the limit $f$. Hence

$$
\begin{aligned}
\mu^{*}(f) & =\lim _{n \rightarrow \infty} \mu^{*}\left(f_{n}\right) \\
& =\lim _{n \rightarrow \infty} \sum a_{x} f_{n}(x) .
\end{aligned}
$$

So once again we get

$$
\mu^{*}(f) \leqq \sum a_{x} f(x)
$$

On the other hand, let $\sum a_{x} f(x)=\sigma \leqq+\infty$. Let $E=\left\{x \in X: a_{x}>0\right\}$. Then

$$
\sum_{x \in E} a_{x} f(x)=\sigma .
$$

If $\sigma^{\prime}<\sigma$, there exists a finite set $F \subset E$ such that

$$
\sum_{x \in F} a_{x} f(x)>\sigma^{\prime} .
$$

If $f$ is finite-valued on $F$, it is clear that

$$
\mu^{*}\left(f_{n}\right) \geqq \sum_{x \in F} a_{x} f_{n}(x)
$$

will exceed $\sigma^{\prime}$ if $n$ is large enough, and then (since $\sigma^{\prime}$ is arbitrary save for the condition $\left.\sigma^{\prime}<\sigma\right) \mu^{*}(f) \geqq \sigma$. So (2.3) is true in this case. Otherwise, there exists $x_{0} \in F$ with $f\left(x_{0}\right)=+\infty$, and then $f_{n}\left(x_{0}\right)=n$. So $\mu^{*}(f) \geqq \mu^{*}\left(f_{n}\right)=\sum a_{x} f_{n}(x)$ $\geqq a_{x_{0}} f_{n}\left(x_{0}\right)=n a_{x_{0}}$ and so, since $a_{x_{0}}>0, \mu^{*}(f)=+\infty$. Thus (2.3) holds in this case too. This completes the proof.

(b) All sets, and therefore all functions, are $\mu$-measurable.

Proof. Thanks to (2.2), if $W$ is any bounded open subset of $X$, we can find a sequence $\left(\mu_{n}\right)$ of atomic measures with finite supports,

$$
\mu_{n}=\sum_{x \in F_{n}} a_{x} \epsilon_{x} \quad\left(F_{n} \subset X \text { finite }\right)
$$

such that

$$
\left|\mu(f)-\mu_{n}(f)\right| \leqq \delta_{n} \cdot\|f\|_{\infty}
$$

for all $f \in K_{+}(X)$ which vanish on $W^{\prime}$, where $\|f\|_{\infty}$ is the maximum modulus of $f$ and where $\delta_{n} \rightarrow 0$ as $n \rightarrow \infty$. For each $n$, (a) shows that the open set $F_{n}^{\prime}$ is 
$\mu_{n}$-negligible; so every finite function $f \geqq 0$ is equal a.e. $\left(\mu_{n}\right)$ to a function in $\varkappa_{+}(X)$, hence is certainly $\mu_{n}$-measurable. This being so, (b) will follow from this more general assertion.

Lemma. Suppose $\mu$ and $\mu_{n}(n=1,2, \cdots)$ are positive measures, and that (2.4) holds for all $f \in K^{+}(X)$ which vanish on $W^{\prime}$ ( $W$ any fixed open subset of $X)$, where $\delta_{n} \rightarrow 0$ as $n \rightarrow \infty$. Any bounded function $f$ which vanishes on $W^{\prime}$ and which is $\mu_{n}$-integrable for each $n$ is then $\mu$-integrable.

Proof of lemma. We may assume that $f \geqq 0$. It is easily seen that the restriction $f \in \mathcal{K}_{+}(X)$ in (2.4) may be removed, the other assumptions standing as in the original and $\mu(f)$ being replaced by $\mu^{*}(f)$ and $\mu_{n}(f)$ by $\mu_{n}^{*}(f)$ : the argument is direct and based merely on the appropriate definitions $[B, p$. 104, Définition 1; p. 109, Définition 3].

Take any bounded $f \geqq 0$, vanishing on $W^{\prime}$ and $\mu_{n}$-integrable for each $n$. Given $\epsilon>0$, choose $n$ so large that $2 \delta_{n}\|f\|_{\infty} \leqq \epsilon / 2$. Since $f$ is $\mu_{n}$-integrable, we can choose $k \in \mathcal{K}_{+}(X)$, vanishing on $W^{\prime}$, so that $\|k\|_{\infty} \leqq\|f\|_{\infty}$ and $\mu_{n}^{*}(|f-k|)$ $\leqq \epsilon / 2$. Then, by (2.4) extended as said,

$$
\left|\mu^{*}(|f-k|)-\mu_{n}^{*}(|f-k|)\right| \leqq \delta_{n}\|f-k\|_{\infty} \leqq 2 \delta_{n}\|f\|_{\infty} \leqq \epsilon / 2
$$

and so $\mu^{*}(|f-k|) \leqq \epsilon / 2+\epsilon / 2=\epsilon$. It follows that $f$ is $\mu$-integrable [B, p. 131, Définition 2].

(c) The equation (2.3) holds for any $f \geqq 0$, finite or not, for which $\mu^{*}(f)$ $<+\infty$.

Proof. By definition,

$$
\mu^{*}(f)=\operatorname{Inf}\left\{\mu^{*}(h): h \text { lower semicontinuous, } h \geqq f\right\},
$$

and this for any $f \geqq 0$. Thus, by virtue of (a),

$$
\mu^{*}(f) \geqq \sum a_{x} f(x)
$$

holds for every $f \geqq 0$. Assuming that $\mu^{*}(f)<+\infty$, we can choose a sequence $\left(h_{n}\right)$ of integrable lower semicontinuous functions such that $h_{n} \geqq h_{n+1} \geqq f$ and $\mu^{*}\left(h_{n}\right) \downarrow \mu^{*}(f)$ as $n \rightarrow \infty$. Then $h=\operatorname{Inf} h_{n}$ is integrable, $h \geqq f$, and by "monotone convergence" we have

$$
\begin{aligned}
\mu^{*}(h-f) & =\lim \mu^{*}\left(h_{n}-f\right) \\
& =\lim \left[\mu^{*}\left(h_{n}\right)-\mu^{*}(f)\right]=0 .
\end{aligned}
$$

Thus $h=f$ a.e. Now (2.5) shows already that, if a set $E \subset X$ is locally negligible, then $a_{x}=0$ for $x \in E$. Thus $h=f$ on a set $A \subset X$, where $A^{\prime}$ is negligible and therefore $a_{x}=0$ for $x \in A^{\prime}$. Thus

$$
\begin{aligned}
\mu^{*}(h) & =\lim \mu^{*}\left(h_{n}\right)=\lim \sum a_{n} h_{n}(x) \\
& =\lim \sum_{x \in A} a_{x} h_{n}(x) .
\end{aligned}
$$


Since $\mu^{*}\left(h_{n}\right)<+\infty$, it follows that $h_{n}(x)<+\infty$ for each $x \in A$ such that $a_{x}>0$. Accordingly, by shrinking $A$ if necessary, we may assume that each $h_{n}$ is finite-valued on $A$.

Given any $\epsilon>0$, there exists a finite set $F \subset A$ such that

$$
\sum_{x \in F^{\prime} \cap_{A}} a_{x} h_{n}(x) \leqq \epsilon,
$$

first for $n=1$ and then a fortiori for $n>1$. Hence

$$
\sum_{x \in A} a_{x} h_{n}(x) \leqq \sum_{x \in F \cap A} a_{x} h_{n}(x)+\epsilon .
$$

Since $h_{n} \downarrow f$ on $A$, it follows that

$$
\begin{aligned}
\sum_{x \in A} a_{x} h_{n}(x) & \leqq \sum_{x \in F \cap A} a_{x} f(x)+2 \epsilon \\
& \leqq \sum a_{x} f(x)+2 \epsilon
\end{aligned}
$$

for $n \geqq n_{0}$. Then

$$
\mu^{*}(f)=\lim _{n \rightarrow \infty} \sum_{x \in A} a_{x} h_{n}(x) \leqq \sum a_{x} f(x)+2 \epsilon
$$

and so, since $\epsilon>0$ is freely chosen,

$$
\mu^{*}(f) \leqq \sum a_{x} f(x) .
$$

This, combined with (2.5), completes the proof.

(d) For any $f \geqq 0$, finite or not,

$$
\sum a_{x} f(x)=\operatorname{Sup}\left\{\mu^{*}(g): g \leqq f, \mu^{*}(g)<+\infty\right\} .
$$

Proof. Let $L$ and $R$ denote the left- and right-hand sides of (2.6). If $g \leqq f$ and $\mu^{*}(g)<+\infty$ we have by (c)

$$
\mu^{*}(g)=\sum a_{x} g(x) \leqq \sum a_{x} f(x)
$$

and so $R \leqq L$. On the other hand we can find a sequence $\left(F_{n}\right)$ of finite sets such that

$$
\sum_{x \in F_{n}} a_{x} f(x) \rightarrow L
$$

Assuming first that $f$ is finite-valued, put

$$
g_{n}(x)=\left\{\begin{array}{cc}
f(x) & \left(x \in F_{n}\right) \\
0 & \left(x \in F_{n}^{\prime}\right) .
\end{array}\right.
$$

It is clear that $g_{n}$ is integrable and $g_{n} \leqq f$. Hence

$$
\mu^{*}\left(g_{n}\right)=\sum a_{x} g_{n}(x)=\sum_{x \in F_{n}} a_{x} f(x) .
$$


Therefore

$$
R \geqq \operatorname{Sup} \mu^{*}\left(g_{n}\right)=\operatorname{Sup} \sum_{x \in F_{n}} a_{x} f(x)=L .
$$

Thus (2.6) is established when $f$ is finite-valued.

In general, represent $f$ as the monotone limit of the finite functions $f_{n}=\operatorname{Inf}(f, n)$. With an obvious notation we have then $R \leqq L$ and $R_{n}=L_{n}$. Hence $R \geqq \lim R_{n}$ is not less than $\lim L_{n}$. So it remains only to prove that $L \leqq \lim L_{n}$. But $L=\sum a_{x} f(x)$, considered as a function of $f$, is plainly lower semicontinuous relative to the topology of simple convergence (being the supremum of continuous finite sums), whence $L \leqq \lim L_{n}$. This completes the proof of $(d)$.

CoROLlary. Equation (2.3) holds for any $f \geqq 0$, finite or not, which vanishes outside some $\sigma$-finite set.

Proof. Suppose that $f \geqq 0$ is zero outside the union of an increasing sequence $\left(E_{n}\right)$ of integrable sets. With the notation of the proof of $(d)$, we need to show that $\mu^{*}(f)=R$; or, what is equivalent, that $\mu^{*}(f) \leqq R$. Now if $g_{n}$ $=\operatorname{Inf}(f, n) \cdot \phi_{E_{n}}$, then $g_{n}$ is integrable and $g_{n} \uparrow f$. By "monotone convergence" therefore

$$
R \geqq \operatorname{Sup} \mu^{*}\left(g_{n}\right)=\mu^{*}(f),
$$

as required.

(e) For any $f \geqq 0$, finite or not, one has

$$
\bar{\mu}^{*}(f)=\sum a_{x} f(x) .
$$

Proof. By definition $\left[\mathrm{B}^{\prime}\right.$, p. 8, Définition 1]

$$
\bar{\mu}^{*}(f)=\operatorname{Sup} \mu^{*}\left(f \phi_{K}\right),
$$

$K$ ranging over the compact subsets of $X$. By the corollary to (d), the righthand side here is

$$
\operatorname{Sup} \sum a_{x} f(x) \phi_{K}(x)=\operatorname{Sup} \sum_{x \in K} a_{x} f(x)
$$

Since the compact sets include all finite sets this is just

$$
\sum a_{x} f(x)
$$

Corollary. $A$ set $E \subset X$ is locally negligible if and only if $a_{x}=0$ for $x \in E$.

2.2. We now return to the problem of the stability of $\overline{\mathcal{L}}^{1}(w ; \mu)$, where the atomic measure $\mu$ is defined by (2.1) and where $w$ is positive and locally integrable, i.e.

$$
\sum_{x \in K} a_{x} w(x)<+\infty
$$


for each compact $K \subset X$. Let us assume that $\overline{\mathscr{L}}^{1}(w ; \mu)$ is stable under the mapping $f \rightarrow f \circ s$ for some $s \in S$, and then try to follow as closely as possible the arguments used in the proof of Proposition 1.11.

Throughout the remainder of this section we shall denote by $E$ the set of $x \in X$ for which $a_{x} w(x)>0$. We write, as before,

$$
\begin{aligned}
N(f) & =\int|f| w d \mu=\bar{\mu}^{*}(|f| w)=\sum a_{x} w(x)|f(x)| \\
& =\sum_{x \in E} a_{x} w(x)|f(x)|
\end{aligned}
$$

and likewise

$$
\begin{aligned}
N^{\prime}(f) & =\int|f \circ s| w d \mu=\sum_{x \in E} a_{x} w(x)|f(s \cdot x)| \\
& =\sum_{x \in s E} a_{s^{-1} \cdot x w\left(s^{-1} \cdot x\right)|f(x)|} \\
& =N_{1}^{\prime}(f)+N_{2}^{\prime}(f),
\end{aligned}
$$

where

$$
\begin{aligned}
& N_{1}^{\prime}(f)=\sum_{x \in E \cap_{8} E} a_{s^{-1} \cdot x} w\left(s^{-1} \cdot x\right)|f(x)|, \\
& N_{2}^{\prime}(f)=\sum_{x \in E^{\prime} \cap_{8} E} a_{s^{-1} \cdot x} w\left(s^{-1} \cdot x\right)|f(x)| .
\end{aligned}
$$

Because of $(2.1, \mathrm{~b}), \overline{\mathfrak{L}}^{1}(w ; \mu)$ is characterized by the condition $N(f)<+\infty$; and $f \circ s \in \overline{\mathcal{L}}^{1}(w ; \mu)$ if and only if $N_{1}^{\prime}(f)<+\infty$ and $N_{2}^{\prime}(f)<+\infty$.

Now any $f$ which vanishes on $E$ belongs to $\overline{\mathfrak{L}}^{1}(w ; \mu)$, so that stability implies that

$$
N_{2}^{\prime}(f)=\sum_{x \in E^{\prime} \cap_{s B}} a_{x^{-1} \cdot x^{2}} w\left(s^{-1} \cdot x\right) f(x)<+\infty
$$

for every $f \geqq 0$ defined on $E^{\prime}$ (and there finite-valued). Since

$$
a_{s^{-1} \cdot x} w\left(s^{-1} \cdot x\right)>0
$$

for each $x \in E^{\prime} \cap s E$, it follows that

$E^{\prime} \cap s E$ is finite.

On the other hand, since the convergence of $f$ in $\overline{\mathscr{L}}^{1}(w ; \mu)$ implies its pointwise convergence on $E$, we see at once that $N_{1}^{\prime}$ is lower semicontinuous and hence continuous (Baire's theorem again). This entails that

$$
\bar{M}(s) \equiv \operatorname{Sup}_{x \in E}\left\{a_{s^{-1} \cdot x} w\left(s^{-1} \cdot x\right) / a_{x} w(x)\right\}<+\infty .
$$

Reciprocally, if (A) and (B) are satisfied, then $N(f)<+\infty$ implies that 
both $N_{1}^{\prime}(f)$ and $N_{2}^{\prime}(f)$ are finite, so that $\overline{\mathscr{L}}^{1}(w ; \mu)$ is then stable under $f \rightarrow f \circ s$. Now $\bar{M}(s)$ is not generally a subcharacter (as was $M(s)$ earlier). However, (A) and (B) together show that

$$
a_{s^{-1} \cdot x} w\left(s^{-1} \cdot x\right) \leqq M \cdot a_{x} w(x)
$$

holds, with $M=M(s)$, except perhaps for a finite set of $x \in X$. Indeed, by (B), this holds for $x \in E$; it holds trivially for $x \in s E^{\prime}$; hence it fails to hold at most on a subset of $\left(E \cup s E^{\prime}\right)^{\prime}=E^{\prime} \cap s E$, which, by (A) is finite. Thus $M(s)$, defined to be the infimum of numbers $M \geqq 0$ for which (2.7) holds, satisfies $M(s)$ $\leqq \bar{M}(s)$. This $M(s)$ is clearly a subcharacter of $S$. The condition $M(s)<+\infty$ is necessary and sufficient for the stability of $\overline{\mathscr{L}}^{1}(w ; \mu)$ under $f \rightarrow f \circ s$. Thus we have the following analogue of Theorem 1.13.

2.3. Theorem. Let $\mu$ be defined by (2.1). In order that $\overline{\mathscr{L}}^{1}(w ; \mu)$ be stable under $S$ it is necessary and sufficient that, for each $s \in S$, a number $M(s) \geqq 0$ shall exist such that the inequality expressed in (1.4') holds save perhaps on a finite subset of $X$ (which may depend on $s$ ). If, in addition, w is not locally negligible, $M(s)$ is a strictly positive subcharacter of $S$ such that $M(1)=1$.

Proof. We had reached (2.7) without assuming relative invariance of $\mu$. This last signifies that $a_{s-1 \cdot x}=\Delta(s) a_{x}$, and then (2.7) transforms at once into $\left(1.4^{\prime}\right)$. The proof is thus complete.

We may remark that the conclusions of Proposition 1.16 are again valid.

3 . The group case. Henceforth we assume that $X$ is a locally compact group, $\mu$ its left Haar measure, and $S$ acts on $X$ by left- or right-translation. The latter case differs from the former only by the presence of a nontrivial modular function, and it will suffice to deal with the case of left-translations, so that $\Delta(s) \equiv 1$.

Only two cases arise, namely:

(1) $X$ is discrete, and then $\overline{\mathscr{L}}^{1}(w ; \mu)=\mathscr{L}^{1}(w ; \mu)$.

(2) $X$ is nondiscrete; then $\mu$ is diffuse and satisfies $\left({ }^{*}\right)\left({ }^{6}\right)$; the stability of $\overline{\mathscr{L}}^{1}(w ; \mu)$ is equivalent to that of $\mathscr{L}^{1}(w ; \mu)$ provided either $P$ or $P^{\prime}$ is $\sigma$-finite (Corollary 1.8; recall that $P=\{x \in X: w(x)>0\}$ ).

In this section we discuss further the properties of a weight-function $w$ which is moderate in the sense of Definition 1.12 , so that $\left(1.4^{\prime}\right)$ holds. We shall show that $w$, if not locally negligible, satisfies certain two-sided inequalities which restrict the rate of growth of both $w$ and $w^{-1}$ in terms of that of the subcharacter $M(s)$. Note that $M(s)$ is subject to the results stated in Propositions 1.15 and 1.16. The inequalities referred to take the form

$$
\begin{aligned}
w(y) / M(y) M\left(x^{-1}\right) & \leqq w(y) / M\left(y x^{-1}\right) \leqq w(x) \leqq w(y) N\left(x y^{-1}\right) \\
& \leqq w(y) M(x) M\left(y^{-1}\right),
\end{aligned}
$$

${ }^{(6)}$ That is, $s \cdot x=s^{-1} x\left(\right.$ or $x s^{-1}$ ) in the sense of the group operation. We shall henceforth write $f_{s}$ in place of $f \circ s$. 
holding for specified sets of $x$ and $y$. If (1.4') holds for all $x \in X,(3.1)$ is an immediate deduction therefrom; this is the case if $w$ is continuous. In general, however, we know merely that (3.1) holds for $x \in N^{\prime}$, where $N$, which may depend on $s$, is locally negligible. Consequently a little more argument is needed to show that (3.1) holds for locally almost all $x$ and locally almost all $y$.

3.1. TheOREM. If $w$ is moderate, there exists a locally negligible set $N$ such that (3.1) holds for $x \in N^{\prime}$ and $y \in N^{\prime}$.

Proof. As we know, the theorem is true with $N=\varnothing$ if $w$ is continuous. To deal with the general case, let us assume for the moment the following proposition $(P)$ : if $A \subset X$ is $\sigma$-compact, there exists a negligible set $A_{0} \subset A$ such that (3.1) holds for $x \in A-A_{0}, y \in A-A_{0}$. From this we derive the required conclusion in the following way. It is known [L, p. 109] that $X$ is the disjoint union of a (generally noncountable) family $\left(X_{i}\right)$ of open-closed, $\sigma$-compact subsets; each $X_{i}$ is a left coset $s V^{\infty}$, where $V$ is a fixed compact and symmetric neighbourhood of $e$, so that each compact in $X$ is covered by finitely many of the $X_{i}$. According to $(P)$, there exists for each $i$ a negligible set $Y_{i} \subset X_{i}$ such that (3.1) holds for $x \in X_{i}-Y_{i}, y \in X_{i}-Y_{i}$. If $L \subset X \times X$ is the set of $(x, y)$ for which $(3.1)$ is false, then $(x, y) \in L$ implies that there exist indices $i$ and $j$ such that $(x, y) \in X_{i} \times X_{j}$ and $(x, y) \notin\left(X_{i}-Y_{i}\right) \times\left(X_{j}-Y_{j}\right)$, and so either $(x, y) \in X_{i} \times X_{j} \subset Y_{i} \times X$ or $(x, y) \in X_{i} \times Y_{j} \subset X \times Y_{j}$ (or both). Let $N=\cup Y_{i}$. Then $(x, y) \in L$ implies that either $x \in N$ or $y \in N$ (or both). So $(x, y) \in N^{\prime} \times N^{\prime}$ implies that (3.1) holds. The theorem will therefore be proved if we can show that $N$ is locally negligible. But if $K \subset X$ is compact, $K$ is contained in finitely many $X_{i}$, say $K \subset X_{i_{1}} \cup \cdots \cup X_{i_{n}}$, and then $N \cap K \subset Y_{i_{1}} \cup \ldots \cup Y_{i_{n}}$ is negligible; thus $N$ is locally negligible, q.e.d.

It remains to prove $(P)$. It is plainly enough to prove $(P)$ in the case in which $A$ is compact. To this end, let $k \in \Re_{+}(X)$ be such that $\int k d \mu=1$; ultimately we shall allow $k$ to converge to the Dirac measure $\epsilon_{e}$, the support of $k$ being a neighbourhood of $e$ which becomes arbitrarily small. For each $k$, let

$$
w_{k}(x)=\int k(y) w(x y) d \mu(y) .
$$

Then $w_{k}$ is continuous and satisfies $\left(1.4^{\prime}\right)$ everywhere, as follows from (1.4') for $w$ itself and the definition of $w_{k}$. Hence (3.1) holds for each $w_{k}$. As $k$ varies, $w_{k} \rightarrow w$ in $\mathscr{L}^{1}(A)$. Hence we may extract a sequence $\left(w_{k}\right)$ which converges to $w$ on $A-A_{0}$, where $A_{0}$ is negligible. $(P)$ then follows by passage to the limit as $n \rightarrow \infty$. The proof is thus complete.

3.2. Remark. As we have said, there is no loss of generality in assuming that $w$ is everywhere finite. It therefore follows that there exist numbers $c$ and $c^{\prime}$ depending only on $w$ such that

$$
c \cdot M\left(x^{-1}\right)^{-1} \leqq w(x) \leqq c^{\prime} \cdot M(x)
$$


1.a.e.; also, if $w$ is not locally negligible, (3.2) holds l.a.e. for some number $c>0$. If, for example, $X$ is the additive group of real numbers, (3.2) shows that for some number $a \geqq 0$ one has

$$
c \cdot \exp (-a|x|) \leqq w(x) \leqq c^{\prime} \cdot \exp (a|x|)
$$

for almost all $x$. Reciprocally, if $w$ satisfies these inequalities, then it is moderate.

3.3. $X$ an infinite compact group. For this case the preceding results yield at once the conclusion that $\mathscr{L}^{1}(w ; \mu)$ is stable under left- (or right-) translations if and only if either (i) $w=0$ a.e., or (ii) both $w$ and $w^{-1}$ are essentially bounded functions. Moreover, if $1 \leqq p<+\infty$, the same is true with $\mathfrak{L}^{p}(w ; \mu)$ replacing $\mathscr{L}^{1}(w ; \mu)$.

The latter assertion, with $p=2$, has direct bearing on the problem of translational bases in the $\mathscr{L}^{2}$-space over a discrete infinite group $G$. In fact, this problem and the convergence questions it raises reduce to the study of the convergence of Fourier series in the space $\mathscr{L}^{2}(w ; \mu)$ constructed from the group $X$ dual to $G, w$ being now a positive integrable function on $X$ such that $w^{-1}$ is also integrable (so that case (i) is excluded from the outset). It is an apparently unsolved problem to determine conditions upon $w$ in order that each $f \in \mathscr{L}^{2}(w ; \mu)$ is the limit, in that space, of its Fourier series. It is now apparent that, unless (ii) holds (in which case $\mathfrak{L}^{2}(w ; \mu)$ is just the familiar $\mathfrak{L}^{2}$-space), there can be no characterization of the Fourier transforms of functions in $\mathcal{L}^{2}(w ; \mu)$ which is expressible solely in terms of the modulus of the Fourier transform. The expected Parseval formula reads

$$
\int|f|^{2} w d \mu=\sum_{u} \sum_{v} w\left(u v^{-1}\right) \hat{f}(u)[\hat{f}(v)]^{-},
$$

where the "hat" ^ denotes the Fourier transform and where $u$ and $v$ run separately over the (infinite discrete) group dual to $X$. Except in the trivial case (ii), it is clear that the series just written cannot converge absolutely for each $f \in \mathscr{L}^{2}(w ; \mu)$, and it would be interesting to know if the suggested Parseval formula is valid for each $f$ with any suitable interpretation of the double sum. Incidentally, it is easily seen (e.g. by use of the Hahn-Banach theorem) that in any case the "trigonometric polynomials" are dense in $\mathcal{L}^{2}(w ; \mu)$.

4. $\bar{L}^{1}(w)$ as a $\$$-algebra. In view of the importance of $\mathscr{L}^{1}$ as an example of an algebra under convolution, and the close relationship of this algebraic structure with translational problems, it is natural to investigate conditions under which $\overline{\mathscr{L}}^{1}(w)$ and/or $\mathscr{L}^{1}(w)$ are $\$$-algebras. In case $X$ is the additive group of real numbers, $\mathcal{L}^{1}(w)$ (or its quotient $L^{1}(w)$ ) has been investigated as a topological algebra under convolution for certain simple choices of $w$ which are continuous, strictly positive subcharacters. The following results help to elucidate to what extent these restrictions on $w$ are practically obligatory. 
4.1. We begin with a few remarks concerning the concept of convolution to be used. If $f$ and $g$ are positive measurable functions, the integral

$$
f \notin g(x)=\int f(y) g\left(y^{-1} x\right) d \mu(y)
$$

exists, possibly $+\infty$, for each $x \in X$. Perpetrating a mild abuse of language, we shall say that $f \star g \in \overline{\mathscr{L}}^{1}(w)$ (resp. $\left.\mathcal{L}^{1}(w)\right)$ if $(f \star g) w$ is equal l.a.e. (resp. a.e.) to a function in $\mathcal{L}^{1}$, it being understood that $(f \notin g) w$ is to be 0 at any point at which $w=0$ and to be $+\infty$ at any point where $f \notin g=+\infty$ and $w>0$.

Unless we know that $w>0$ holds l.a.e. (resp. a.e.), it is not possible to extend this definition satisfactorily to the case in which $f$ and $g$ are no longer necessarily positive. It is at this point that the significance of Proposition 4.2 below becomes apparent.

Meanwhile, assuming that $w>0$ l.a.e. (resp. a.e.), if $f$ and $g$ are not necessarily positive, we say that $f \notin g \overline{\mathscr{L}}^{1}(w)$ (resp. $\mathscr{L}^{1}(w)$ ) if the same is true of $|f| \notin|g|$. Then there exists at least one (actually many) decompositions $f=f_{1}-f_{2}, g=g_{1}-g_{2}$ in which the $f_{i}$ and $g_{i}$ are positive and $f_{i} \star g_{j} \in \bar{L}^{1}(w)$ (resp. $\mathscr{L}^{1}(w)$ ) for $i, j=1,2$; each $f_{2} \nless g_{j}$ is then finite-valued l.a.e. (resp. a.e.) and we may define $f \notin g$ l.a.e. (resp. a.e.) by

$$
f \notin g=f_{1} \star g_{1}-f_{1} \star g_{2}-f_{2} \star g_{1}+f_{2} \star g_{2} \text {. }
$$

It is then clear that for a given $f$ (resp. $g$ ), the set of $g$ (resp. $f$ ) such that $f \star g \in \overline{\mathscr{L}}^{1}(w)$ (resp. $\mathscr{L}^{1}(w)$ ) is linear. Also $f \notin g$, whenever it exists in the above sense, depends only on the classes (modulo locally negligible functions) defined by $f$ and $g$, whilst $f \notin g$ defines a unique such class depending bilinearly on the pair $(f, g)$.

Here now is the analogue of Proposition 1.5.

4.2. Proposition. If $X$ is nondiscrete, and if $f \notin g \in \overline{\mathcal{L}}^{1}(w)$ whenever $f \geqq 0$ is in $\mathscr{L}^{1}(w)$ and $g \geqq 0$ is bounded and each has a bounded support, then EITHER $w=0$ l.a.e. $O R w>0$ l.a.e.

Proof. If $w$ is not l.a.e. strictly positive, there exists a compact set $A \subset X$ with $\mu(A)>0$ and $w=0$ on $A$. Then any measurable $f \geqq 0$ vanishing outside $A$ belongs to $\mathscr{L}^{1}(w)$, and so $f \notin g \in \overline{\mathscr{L}}^{1}(w)$ for each $g$ of the specified type. This signifies that

$$
\int w(x) d \mu(x) \int_{A} f(y) g\left(y^{-1} x\right) d \mu(y)<+\infty
$$

for such $f$ and $g$.

If it were at the same time false to assert that $w=0$ 1.a.e., there would exist a compact set $B \subset X$ such that $\mu(B)>0$ and $w(x) \geqq c>0$ for $x \in B, c$ being a suitable number. A contradiction would result as soon as $f$ and $g$ were produced satisfying 


$$
\int_{A} f(y) g\left(y^{-1} x\right) d \mu(y)=+\infty
$$

for all $x \in B$. But for this it suffices to take for $g$ the characteristic function of the compact set $A^{-1} B$, and then construct $f$ as follows. $X$ being nondiscrete, there exists a sequence $\left(A_{n}\right)$ of disjoint integrable subsets of $A$ such that $\mu\left(A_{n}\right)>0$ for each $n$. Let then $f=\mu\left(A_{n}\right)^{-1}$ on $A_{n}(n=1,2, \cdots)$ and $f=0$ elsewhere. This will give for $x \in B$

$$
\begin{aligned}
\int_{A} f(y) g\left(y^{-1} x\right) d \mu(y) & =\sum_{n} \mu\left(A_{n}\right)^{-1} \int_{A_{n}} g\left(y^{-1} x\right) d \mu(y) \\
& =\sum_{n} \mu\left(A_{n}\right)^{-1} \cdot \mu\left(A_{n}\right)=+\infty
\end{aligned}
$$

since $g\left(y^{-1} x\right)=1$ for $y \in A, x \in B$. This completes the proof.

Before proceeding to the main theorem of this section, we make two remarks. It is first of all obvious that if $w=0$ 1.a.e., then $\overline{\mathscr{L}}^{1}(w)$ and $\mathcal{L}^{1}(w)$ are both $\Varangle$-algebras. Next, if either of these structures is known to be a $\Varangle$-algebra, it is sometimes possible to infer that the same is true of the other by use of the following result.

4.3. Proposition. (i) If $\mathfrak{L}^{1}(w)$ is a $\$$-algebra, so too is $\overline{\mathscr{L}}^{1}(w) \cdots$ in fact $\overline{\mathscr{L}}^{1}(w) \notin \overline{\mathscr{L}}^{1}(w) \subset \mathcal{L}^{1}(w)$.

(ii) If $\overline{\mathscr{L}}^{1}(w)$ is a -algebra, and if either $P$ or $P^{\prime}$ is $\sigma$-finite, then $\mathscr{L}^{1}(w)$ is a $\$$-algebra.

Proof. We may suppose throughout that $f$ and $g$ are positive and that $X$ is nondiscrete. (The two spaces coincide if $X$ is discrete.)

(i) By Proposition 4.2 we may assume that $w>0$ 1.a.e. Then, if $f$ and $g$ belong to $\overline{\mathscr{L}}^{1}(w)$, there exist $f^{\prime}$ and $g^{\prime}$ in $\mathscr{L}^{1}(w)$ such that $f=f^{\prime}$ and $g=g^{\prime}$ 1.a.e. But then $f \notin g=f^{\prime} \star g^{\prime}$ everywhere, and so $f \notin g \in \mathscr{L}^{1}(w)$.

(ii) Let $f, g \in \mathcal{L}^{1}(w)$. There is then a $\sigma$-finite set $E$ outside which both $f w$ and $g w$ vanish, so that $f$ and $g$ vanish outside $P^{\prime} \cup E$, and therefore $f \notin g$ vanishes outside $\left(P^{\prime} \cup E\right)^{2}$. Therefore $(f \notin g) w$ vanishes outside $P \cap\left(P^{\prime} \cup E\right)^{2}$. Since, by hypothesis, $f \notin g \in \overline{\mathcal{L}}^{1}(w)$, it suffices to show that $P \cap\left(P^{\prime} \cup E\right)^{2}$ is $\sigma$-finite. If we assume for the moment that $H^{2}$ is $\sigma$-finite whenever $H$ is $\sigma$ finite, it is clear that $P \cap\left(P^{\prime} \cup E\right)^{2}$ is $\sigma$-finite if either of $P$ or $P^{\prime}$ has this property.

Suppose then that $H$ is $\sigma$-finite. It is then contained in the union of a sequence $\left(A_{i}\right)$ of open integrable sets, and $H^{2}$ is contained in the union of the $A_{i} A_{j}$. So it suffices to show that $A B$ is $\sigma$-finite whenever $A$ and $B$ are open and integrable. Now $\phi_{A} \sharp \phi_{B}(x)=\mu\left(A \cap x B^{-1}\right)$, and this is nonzero if and only if $x \in A B$. Since $\mathcal{L}^{1}$ is itself a $\$$-algebra, this last condition holds at most for a $\sigma$-finite set of $x$. Thus $A B$ is $\sigma$-finite. This completes the proof.

We pass now to the main theorem of this section. 
4.4. Theorem. (I) Suppose that $w>0$ l.a.e. and is essentially bounded on compact sets. If $\overline{\mathfrak{L}}^{1}(w)$ is a $\$$-algebra, then it is translation invariant and there is a number $C$ such that

$$
N(f \notin g) \leqq C \cdot N(f) N(g)
$$

$\left(f, g \in \overline{\mathscr{L}}^{1}(w)\right)$.

If in addition w is upper semicontinuous, and if $M$ is the subcharacter corresponding to $w$ as in $\$ 1$, then $w$ is equivalent to $M$ in the sense that there exist numbers $k$ and $k^{\prime}$ such that $0<k \leqq k^{\prime}<+\infty$ and $\left({ }^{7}\right)$

$$
k \cdot M(s) \leqq w(s) \leqq k^{\prime} \cdot M(s) .
$$

l.a.e.

(II) If $w$ is equivalent to a subcharacter, then $\mathscr{L}^{1}(w)$ is simultaneously translation-invariant and a -algebra, the same is true of $\overline{\mathcal{L}}^{1}(w)$, and (4.1) holds for a suitable number $C$.

Proof. $A d(\mathrm{I})$. Since $w>0$ 1.a.e., for a given $f \in \overline{\mathfrak{L}}^{1}(w)$ we may regard $T: g \rightarrow f \notin g$ as a linear map of $\bar{L}^{1}(w)$ into itself. (We take the license of making no notational distinction between a function and the class it defines.) We shall show that $T$ has a closed graph, i.e. that if $g_{n} \rightarrow 0$ in $\bar{L}^{1}(w)$ and $h_{n}=f \notin g_{n}$ $\rightarrow h$ in $\bar{L}^{1}(w)$, then the function $h$ is necessarily 0 l.a.e. (which signifies that the class $h$ is 0$)$. To see this we notice first that if $k \in K_{+}(X)$ then

$$
\int k\left|h_{n}\right| d \mu \leqq \int k\left(|f| \nless\left|g_{n}\right|\right) d \mu .
$$

On the other hand, by passing to a suitable subsequence if necessary, we may assume that $g_{n} \rightarrow 0$ and $h_{n} \rightarrow h$ l.a.e. The modified form of Fatou's Lemma then gives

$$
\begin{aligned}
\int k|h| d \mu & \leqq \liminf _{n \rightarrow \infty} \int k\left|h_{n}\right| d \mu \\
& \leqq \liminf _{n \rightarrow \infty} \int k\left(|f| \notin\left|g_{n}\right|\right) d \mu
\end{aligned}
$$

and

$$
\begin{aligned}
\liminf _{n \rightarrow \infty}|f| \bowtie\left|g_{n}\right|(x) & =\liminf _{n \rightarrow \infty} \int|f(y)| \cdot\left|g_{n}\left(y^{-1} x\right)\right| d \mu(y) \\
& \leqq \int|f(y)| \liminf _{n \rightarrow \infty}\left|g_{n}\left(y^{-1} x\right)\right| d \mu(y)=0 .
\end{aligned}
$$

(7) The right-hand inequality holds l.a.e. without the restriction of upper semicontinuity on $w$, and at the same time the left-hand inequality may be replaced by lim ess $\sup _{x \rightarrow \mathbf{s}} w(x)$ $\geqq k \cdot M(s)$ for all $s \in X$. Note that, with the notation of the proof to follow, lin ess $\sup _{x \rightarrow s} w(x)$ $\geqq \lim \sup _{i} \int f_{i} w d \mu$; if $w$ is upper semicontinuous, $w(s)=\lim \sup _{x \rightarrow s} w(x) \geqq \lim \operatorname{ess}_{\sup _{x \rightarrow s}} w(x)$ and we recover the left-hand inequality in (4.2), actually holding everywhere. 
Consequently by the same token one has

$$
\liminf _{n \rightarrow \infty} \int k\left(|f| \star\left|g_{n}\right|\right) d \mu \leqq \int k \cdot \liminf _{n \rightarrow \infty}\left(|f| \star\left|g_{n}\right|\right) d_{\mu}=0 .
$$

Thus $\int k|h| d \mu=0$ for all $k \in \Re_{+}(X)$, and so $h=0$ l.a.e., the desired conclusion.

Since $\bar{L}^{1}(w)$ is a Banach space $\left(^{8}\right)$ and $T$ is closed, it follows that $T$ is continuous. Thus for each $f \in \overline{\mathscr{L}}^{1}(w)$ there exists a number $C_{f}$ such that $N(f \notin g) \leqq C_{f} \cdot N(g)$ for all $g \in \overline{\mathscr{L}}^{1}(w)$; in particular, $N(f \notin g)$ is continuous in each argument separately. From this it appears that the seminorm $f \rightarrow \operatorname{Sup}\{N(f \notin g): N(g)=1\}$ is lower semicontinuous on $\overline{\mathfrak{L}}^{1}(w)$. Since the latter space is complete, this seminorm is continuous. Thus (4.1) is established.

This being so, let $s \in X$ and let $\left(f_{i}\right)$ be a directed family in $\varkappa_{+}(X)$ which converges vaguely to the Dirac measure $\epsilon_{s}$ (mass +1 at $s$ ): thus $\int f_{i} d \mu=1$ and $f_{i}$ vanishes outside a neighbourhood of $s$ which becomes arbitrarily small as $i$ increases. Since $w$ is essentially bounded on compact sets, we may arrange that the $f_{i}$ remain bounded in $\overline{\mathscr{L}}^{1}(w)$. Now $\mathcal{K}(X)$ is dense in $\overline{\mathscr{L}}^{1}(w)$, as is to be proved in Lemma 4.5 below, and $\lim _{i} f_{i} \nless g=g_{s}$ in $\overline{\mathscr{L}}^{1}(w)$ whenever $g \in \mathcal{K}(X)$. From (4.1) it then follows that the directed family $\left(f_{i} \star g\right)$ is Cauchy for each $g \in \overline{\mathfrak{L}}^{1}(w)$. So, since $\overline{\mathscr{L}}^{1}(w)$ is complete, this directed family converges in $\overline{\mathscr{L}}^{1}(w)$ to some function which, as is easily seen, can be none other than $g_{s}$. This establishes the claim that $\overline{\mathscr{L}}^{1}(w)$ is translation-invariant.

Introduce the subcharacter $M$ associated with $w$. As we know,

$$
M(s)=\operatorname{Sup}\left\{N\left(f_{s}\right): N(f)=1\right\} .
$$

Accordingly, given $s \in X$ and $\epsilon>0$, we can choose $g \in \overline{\mathfrak{L}}^{1}(w)$ with $N(g)=1$ and $N\left(g_{s}\right) \geqq M(s)-\epsilon$. Since $g_{s}=\lim \left(f_{i} \notin g\right)$, it follows that $N\left(f_{i} \notin g\right) \geqq M(s)-2 \epsilon$ for all sufficiently large $i$. A fortiori

$$
C \cdot N\left(f_{i}\right) N(g) \geqq M(s)-2 \epsilon,
$$

and therefore

$$
\int f_{i} w d \mu \geqq C^{-1}(M(s)-2 \epsilon)
$$

for such $i$. So

$$
M(s) \leqq C \cdot \liminf _{i} \int f_{i} w d \mu .
$$

If $w$ is upper semicontinuous, the lim inf on the right does not exceed $w(s)$, so that $w(s) \geqq C^{-1} \cdot M(s)$. The proof of $(\mathrm{I})$ is completed by appeal to Theorem 3.1.

${ }^{(8)} \bar{L}^{1}(w)$ is the quotient of $\overline{\mathscr{L}}^{1}(w)$ modulo the closed vector subspace $\operatorname{Ker} N$ (i.e., in this case, the subspace formed of locally negligible functions). Since $\overline{\mathscr{L}}^{1}(w)$ is complete (see proof of Proposition 1.11), the same is true of $\bar{L}^{1}(w)$. 
$A d$ (II). If $w$ is equivalent to $M$, then

$$
\overline{\mathscr{L}}^{1}(w)=\overline{\mathscr{L}}^{1}(M) \text { and } \mathscr{L}^{1}(w)=\mathscr{L}^{1}(M) .
$$

So we may as well assume that $w$ is a subcharacter. Then either $w>0$ everywhere or $w=0$ everywhere. According to Propositions 1.7 and 4.3 , if $\overline{\mathscr{L}}^{1}(w)$ is translation invariant (resp. a $\Varangle$-algebra), then so too is $\mathcal{L}^{1}(w)$ translationinvariant (resp. a $\$$-algebra). Thus it suffices to consider throughout the case of $\bar{L}^{1}(w)$, where $w$ is a subcharacter and $w>0$ everywhere (the case $w \equiv 0$ being trivial).

It is at once clear that $\overline{\mathfrak{L}}^{1}(w)$ is translation-invariant, and that $N\left(f_{\mathrm{s}}\right)$ $\leqq w(s) \cdot N(f)$. Moreover, as we shall show in a moment, $f \notin g$ is equal 1.a.e. to the element of $\overline{\mathfrak{L}}^{1}(w)$ represented by the abstract integral

$$
[f, g]=\int f(s) g_{s} d \mu(s)
$$

more precision is added to this statement in the discussion to follow. Assuming this temporarily, it becomes clear that $\overline{\mathscr{L}}^{1}(w)$ is a $\$$-algebra and that

$$
\begin{aligned}
N(f \star g) & =N([f, g]) \leqq \int N\left(\left(f^{\prime}(s) g_{\S}\right)\right) d \mu(s) \\
& \leqq \int w(s)|f(s)| N(g) d \mu(s)=N(f) \cdot N(g),
\end{aligned}
$$

so that the proof of (II) will be completed.

We now examine the abstract integral just introduced. It will be shown in Lemma 4.6 to follow that the function $s \rightarrow g_{s}$, mapping $X$ into $\overline{\mathfrak{L}}^{1}(w)$, is continuous. Since $N\left(f(s) g_{s}\right) \leqq|f(s)| w(s) N(g)$ and $f \in \overline{\mathcal{L}}^{1}(w)$, we can be certain there exists at least one $h \in \overline{\mathscr{L}}^{1}(w)$ satisfying the requirements of a weak (even a Bochner) integral of the function $s \rightarrow f(s) g_{s}$ with values in $\overline{\mathscr{L}}^{1}(w)$, this $h$ being characterized modulo locally negligible functions by the requirement that

$$
F(h)=\int f(s) F\left(g_{s}\right) d \mu(s)
$$

holds for each continuous linear form $F$ on $\overline{\mathfrak{L}}^{1}(w)\left({ }^{9}\right)$. The class of $[f, g]$ depends bilinearly on those of $f$ and $g$, and the same is true of the class of $f \notin g$. So, to identify these classes in all cases, it suffices to consider the case in which $f$ and $g$ are positive. We may also impose the further restriction that $f$ and $g$ vanish outside bounded sets: for, since $w>0$ everywhere, there are monotone increasing sequences $\left(f_{n}\right)$ and $\left(g_{n}\right)$, each $f_{n}$ and each $g_{n}$ vanishing outside a bounded set, such that $f_{n}$ (resp. $g_{n}$ ) converges to $f$ (resp. $g$ ). Then, by the

(?) If desired, the quotient space $\bar{L}^{1}(w)$ may be used throughout; the integral is then a unique element of this separated space. 
principle of dominated convergence, $f_{n} \rightarrow f$ (resp. $g_{n} \rightarrow g$ ) in $\overline{\mathscr{L}}^{1}(w)$ and so, since $[f, g]$ is continuous in the pair of variables, $\left[f_{n}, g_{n}\right] \rightarrow[f, g]$ in $\overline{\mathscr{L}}^{1}(w)$. On the other hand, thanks to monotone convergence, $f_{n} \notin g_{n} \uparrow f \notin g$ everywhere. So the equality $[f, g]=f \notin g$ l.a.e. will follow if it is known that $\left[f_{n}, g_{n}\right]=f_{n} \notin g_{n}$ 1.a.e. for each $n$.

Suppose therefore that $f$ and $g$ are positive and vanish outside bounded sets. To verify that $[f, g]=f \notin g$ l.a.e., it suffices to show that

$$
\int[f, g] b w d \mu=\int(f \star g) b w d \mu
$$

for each measurable $b$ which is bounded and vanishes outside a bounded set. The mapping $h \rightarrow \int h b w d \mu$ is a continuous linear form on $\bar{L}^{1}(w)$ (trivial converse of Lemma 4.5) and so, by very definition of the abstract integral $[f, g]$, we have

$$
\int[f, g] b w d \mu=\int f(s) d \mu(s) \int g\left(s^{-1} x\right) b(x) w(x) d \mu(x) .
$$

Since $f, g$ and $b$ vanish outside bounded sets we may certainly apply the Lebesgue-Fubini Theorem [B', p. 95, Théorème 1] to get

$$
\int[f, g] b w d \mu=\int b(x) w(x) d \mu(x) \int f(s) g\left(s^{-1} x\right) d \mu(s) .
$$

The inner integral is precisely $f \notin g(x)$, and so the righthand side is just $\int(f \star g) b w d \mu$ and we have reached the desired conclusion.

It remains only to prove the two lemmas. Regarding Lemma 4.5 we may note that to assert that $\mathscr{K}=\mathscr{K}(X)$ is dense in $\overline{\mathscr{L}}^{1}(w)$ is equivalent to asserting that it is dense in $\mathscr{L}^{1}(w)\left({ }^{10}\right)$. We deal with the latter space. The required density assertion follows on combining Lemma 4.5 with the Hahn-Banach theorem in an obvious way.

4.5. Lemma. If $F$ is a continuous linear form on $\mathfrak{L}^{1}(w)$, there exists a bounded measurable function $b$ such that

$$
F(f)=\int f b w d \mu
$$

for $f \in \mathscr{L}^{1}(w)$.

Proof. Let $P=\{x \in X: w(x)>0\}$. Continuity of $F$ means that there exists a number $C \geqq 0$ such that

$$
|F(f)| \leqq C \cdot N(f)=C \cdot \int|f| w d \mu
$$

(10) The proof of Proposition 1.7 shows that if $f \in \overline{\mathscr{L}}^{1}(w)$ there exists $g \in \mathcal{L}^{1}(w)$ such that fw $=g w$ l.a.e.; then $\int|f-k| w d \mu=\int|g-k| w d \mu$ for any $k \in \mathcal{K}$. 
for $f \in \mathcal{L}^{1}(w)$. Let $A$ be any bounded integrable set. If $f \in \mathscr{L}^{1}(w)$ vanishes on $A^{\prime},(4.4)$ gives

$$
|F(f)| \leqq C \cdot\left\{\int|f|^{2} w d \mu\right\}^{1 / 2} \cdot\left\{\int_{A} w d \mu\right\}^{1 / 2}
$$

the last factor on the right-hand side is finite since $w$ is locally integrable. The set of $f \in \mathscr{L}^{1}(w)$ which vanish on $A^{\prime}$ and for which $\int|f|^{2} w d \mu<+\infty$ forms a complete (possibly nonseparated) Hilbert space, and the last inequality shows that the restriction of $F$ to this space is continuous. It follows, on passage to the separated quotient space and appeal to elementary results about Hilbert space, that there is a function $b_{A}$ vanishing on $A^{\prime}$ and satisfying $\int\left|b_{A}\right|^{2} w d \mu<+\infty$ for which

$$
F(f)=\int f b_{A} w d \mu
$$

holds for $f$ in the said Hilbert space. Then (4.4) shows that $\left|b_{A}\right| \leqq C$ holds a.e. on $A \cap P$. We may modify $b_{A}$ so as to satisfy $\left|b_{A}\right| \leqq C$ everywhere and $b_{A}=0$ on $(A \cap P)^{\prime}$, neither change affecting the last equation displayed. Thus this last equation holds at least for all bounded functions $f$ which vanish on $A^{\prime}$. Any $f$ in $\mathscr{L}^{1}(w)$ vanishing on $A^{\prime}$ is the monotone limit of a sequence of bounded functions vanishing on $A^{\prime}$, this sequence also converging to $f$ in $\mathfrak{L}^{1}(w)$. Then, by continuity of $F$ and the principle of monotone convergence, our equation continues to hold for each $f$ in $\mathscr{L}^{1}(w)$ which vanishes on $A^{\prime}$.

Now introduce again the partition $\left(X_{i}\right)$ of $X$ used in the proof of Theorem 3.1. The existence of such a partition shows that $X$ may be represented as the union of a family $\left(A_{j}\right)$ of disjoint bounded integrable sets, each compact set being covered by finitely many $A_{j}$. Let $b_{j}$ be $b_{A}$ for $A=A_{j}$. These $b_{j}$ may be pieced together to give a measurable function $b$ satisfying $|b| \leqq C$ everywhere.

If $f \in \mathscr{L}^{1}(w)$, then $f w=0$ a.e. outside a $\sigma$-compact set, and so

$$
f w=\sum_{j}\left(f w \phi_{j}\right)
$$

a.e.,

where $\phi_{j}$ is the characteristic function of $A_{j}$; only a countable number of nonzero terms appear in this sum, and the series converges dominatedly. Thus

$$
f=\sum_{j}\left(f \phi_{j}\right)
$$

in $\mathfrak{L}^{1}(w)$. By continuity of $F$, therefore, we have

$$
\begin{aligned}
F(f)=\sum_{j} F\left(f \phi_{j}\right) & =\sum_{j} \int\left(f \phi_{j}\right) b_{j} w d \mu \\
& =\sum_{j} \int\left(f \phi_{j}\right) b w d \mu .
\end{aligned}
$$


But

$$
f b w=\sum_{j}\left(f \phi_{j}\right) b w
$$

the series converging dominatedly, and so

$$
\int f b w d \mu=\sum_{j} \int\left(f \phi_{j}\right) b w d \mu=F(f) .
$$

This completes the proof.

To establish the fact, used in the proof of Theorem 4.4, (II), that $s \rightarrow f$. is continuous, we need only consider the case in which $w>0$ everywhere. So the following lemma is amply sufficient.

4.6. Lemмa. Suppose that $w>0$ l.a.e. and that $\overline{\mathfrak{L}}^{1}(w)$ is translation-invariant. Then, for each $f \in \overline{\mathcal{L}}^{1}(w)$, the mapping $s \rightarrow f_{\mathrm{s}}$ (from $X$ into $\left.\overline{\mathfrak{L}}^{1}(w)\right)$ is locally uniformly continuous.

Proof. Theorem 1.13 and Proposition 1.16 are available, so that $N\left(g_{8}\right)$ $\leqq M(s) \cdot N(g)$ for $g \in \overline{\mathscr{L}}^{1}(w), M$ being locally bounded. Thus $N\left(f_{t s}-f_{s}\right)$ $\leqq M(s) \cdot N\left(f_{t}-f\right)$ and it suffices to establish continuity at the neutral element $e$.

If we remember that $w$ is locally integrable, it is easily seen that continuity obtains if $f \in \mathcal{K}$.

Given $f \in \overline{\mathcal{L}}^{1}(w)$ and $\epsilon>0$ we may, by Lemma 4.5 and its corollary (that $\Re$ is dense in $\left.\overline{\mathscr{L}}^{1}(w)\right)$, choose $k \in \mathscr{K}$ so that $N(f-k) \leqq \epsilon$. Then

$$
\begin{aligned}
N\left(f_{s}-f\right) & \leqq N\left(f_{s}-k_{s}\right)+N\left(k_{z}-k\right)+N(f-k) \\
& \leqq(1+M(s)) \epsilon+N\left(k_{s}-k\right) .
\end{aligned}
$$

Since $M$ is locally bounded, we see that $s \rightarrow f$, is the limit, uniformly on compact sets, of continuous functions $s \rightarrow k_{s}$. Whence the required result.

\section{REFERENCES}

B. N. Bourbaki, Eléments de mathématique, XIII, Intégration, Chapters I-IV, Paris, 1952. $\mathrm{B}^{\prime}$. - Eléments de mathématique, XXI, Intégration, Chapter V, Paris, 1956.

L. L. H. Loomis, An introduction to abstract harmonic analysis, New York, 1953.

W. A. Weil, L'intégration dans les groupes topologiques et ses applications, 2d ed., Paris, 1951.

UNIVERSITY OF LONDON, LONDON, ENGLAND 